\title{
COSMOLOGY WITHOUT GENERAL RELATIVITY
}

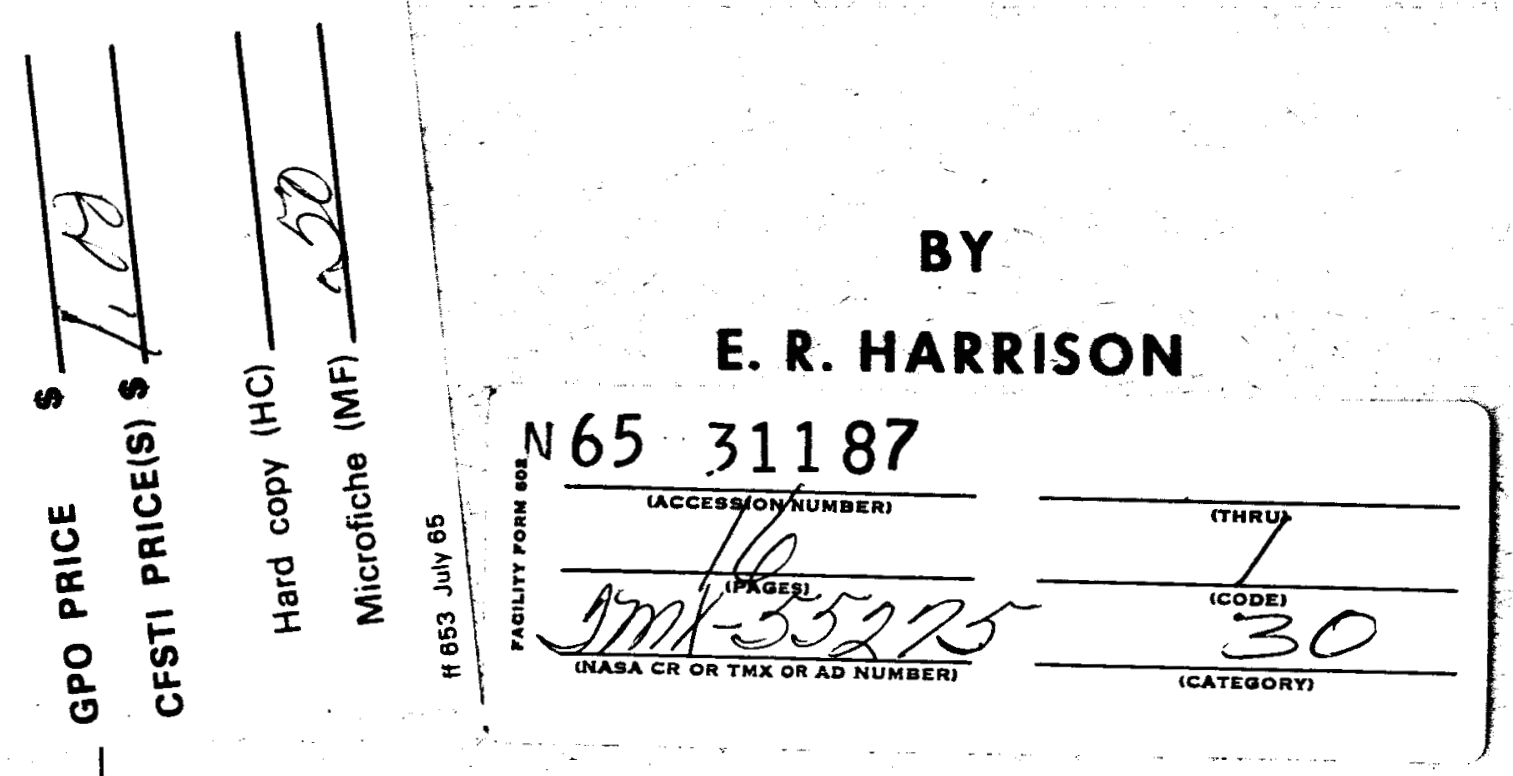

JULY 1965

NASA

GODDARD SPACE FLIGHT CENTER

GREENBELT, MARYLAND 


\section{COSMOLOGY WITHOUT GENERAL RELATIVITY}

E. R. Harrison ${ }^{* *}$

Theoretical Division

July 9, 1965

\section{National Aeronautics and Space Adminstration Goddard Space Flight Center \\ Greenbelt, Maryland}

†National Academy of Sciences-National Research Council Resident Research Associate. * On leave from the Rutherford High Energy Laboratory, Chilton, Didcot, Berkshire, England. 
The usual idealizations in cosmology, which are used with the general theory of relativity for deriving the cosmological equations, lead to the idea of a partitioned universe and to the boundary conditions necessary for isolating a small region or cell of space. An isolated cell, infinitesimally small and embedded in flat space, forms the basis of a microcosmic model of the universe. An internal observer perceives an expanding unbounded universe and applies the same theoretical concepts commonly used in macrocosmic models. He is also free to work within the framework of special relativity theory, and from the first law of thermodynamics and the equations of hydrodynamics is able to derive the cosmological equations without the aid of general relativity and without making any approximations. The application of the cosmological principle to a universe containing a uniform perfect fluid accounts for the complete equivalence of the microcosmic model with the usual macrocosmic models. In many of its properties the microcosmic model closely resembles Newtonian cosmology; it avoids however the ambiguous nature of the gravitational field in a uniform unbounded fluid and is also not limited in its treatment to a pressure-free universe. 


\section{CONTENTS}

Page

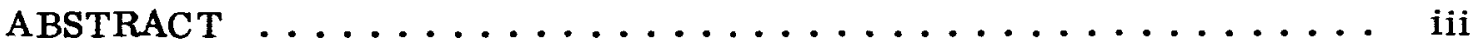

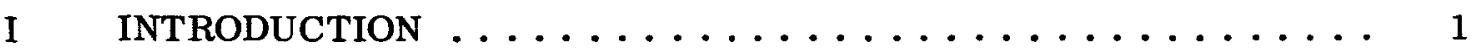

II THE COSMOLOGICAL PRINCIPLE AND

GENERAL RELATIVITY ...................... 2

III A PARTITIONED UNIVERSE .................... 3

IV THE MICROCOSMIC MODEL ................... 4

V NEWTONIAN COSMOLOGY .................... 9

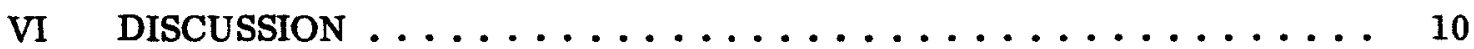

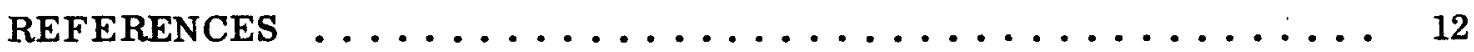




\section{COSMOLOGY WTTHOUT GENERAL RELATIVITY}

\section{INTRODUCTION}

The cosmological equations are normally and properly derived from general relativity theory with the aid of the cosmological principle. The possible models of the universe are then classified according to whether the curvature constant of the metric is positive, zero, or negative.

In 1934, McCrea and Milne used Newtonian theory to derive the cosmological equations for a universe in which the pressure is zero. Although a Newtonian treatment is not entirely free from ambiguity $2,3,4$ it is nevertheless valuable for revealing the implicit simplicity of the cosmological equations. The success of McCrea and Milne's work raises the question: what adaptation or extension of the Newtonian treatment is needed in the more general case of a non-zero pressure? (without, of course, the help of supplementary theorems from general relativity, as in Reference 5). It is assumed that the universe contains a perfect fluid and is homogeneous and isotropic at every point in space. The idea of a partitioned universe then leads to the boundary conditions necessary for isolating a small region or cell of space. Such a cell, infinitesimally small, isolated and embedded in flat space, forms the basis of a microcosmic model of the universe. When the fluid is pressure-free the model obeys the Newtonian equations accurately. This method avoids the ambiguous nature of the gravitational field in a uniform and infinitely extended Newtonian universe. By working within the framework of special relativity we furthermore obtain the cosmological equations in the more general case of a non-zero pressure.

It is emphasized that the general theory of relativity provides the most elegant and effective approach to cosmology, and the approach outlined in this discussion does not pretend in any way to supplant its superior position. The present approach offers insight into the physical nature of the cosmological equations and of the idealizations on which they are founded.

In Section II the cosmological principle is considered briefly and also the results from general relativity for an idealized universe. In Section III the notion of a partitioned universe is outlined and this leads in Section IV to a study of the microcosmic model. Some comparisons and comments are made in Section $\mathrm{V}$ concerning Newtonian cosmology and the physics of the microcosmic model are discussed in Section VI. 


\section{THE COSMOLOGICAL PRINCIPLE AND GENERAL RELATIVITY}

If space has uniform curvature the line-element is

$$
d s^{2}=c^{2} d t^{2}-\frac{R^{2}(t)}{\left(1+\frac{1}{4} k r^{2}\right)^{2}}\left(d r^{2}+r^{2} d \theta^{2}+r^{2} \sin ^{2} \theta d \phi^{2}\right)
$$

In this metric intervals of cosmic time $t$ are measured along world-lines orthogonal to a spatial hypersurface of uniform curvature which is mapped with $r, \theta, \phi$ comoving coordinates. The curvature constant is $\mathrm{k}=0, \pm 1$.

The cosmological principle states that at any instant of cosmic time the universe appears the same for all comoving observers. Generally, the local environment of an observer is irregular. Matter tends to aggregate into objects of stellar and galactic size and is rarely distributed uniformly in relatively small regions of space. The average energy density $\epsilon(r, \theta, \phi)$ in a region of volume $\mathrm{V}$ is

$$
\langle\epsilon\rangle=\mathrm{V}^{-1} \int \epsilon(\mathrm{r}, \theta, \phi) \mathrm{dV}
$$

The cosmological principle is a meaningful postulate when

$$
\langle\epsilon\rangle \rightarrow \epsilon(\mathrm{t})+\delta \epsilon, \text { as } \mathrm{V} \rightarrow \mathrm{U}
$$

where $\delta \epsilon$ is a negligible quantity, $\mathrm{U}$ is everywhere small in comparison with the observable universe, and $\epsilon(t)$ is the average energy density of the universe. The small-scale irregularities are smoothed out as $\mathrm{V}$ increases and eventually $\langle\epsilon>$ becomes $\epsilon(t)$ and the centre of mass of $U$ is stationary in the comoving coordinate system.

Instead of the actual universe it is usual to consider an idealized universe that is isotropic and homogeneous at all points in conformity with the Robertson lineelement (Equation 1), and contains a uniform perfect fluid of proper energy density $\epsilon(t)$ and isotropic pressure $p(t)$. The energy-momentum tensor $T_{j}^{i}$ for the perfect 
fluid in an idealized universe is

$$
\mathbf{T}_{0}^{0}=\epsilon, \mathbf{T}_{1}^{1}=\mathbf{T}_{2}^{2}=\mathbf{T}_{3}^{3}=-\mathbf{p}
$$

The line-element (Equation 1) and the energy-momentum tensor (Equation 3) are geometrical and kinematical interpretations of the cosmological principle, and all that remains is to determine $R(t)$ in Equation (1) with the general relativity equation

$$
R_{j}^{i}-\frac{1}{2} g_{j}^{i} R_{k}^{k}=-\frac{8 \pi G}{c^{2}} T_{j}^{i}
$$

where $G$ is the gravitational constant.

The solution of this equation, given (Equation 1) and (Equation 3), is

$$
\frac{8 \pi \mathrm{G}}{c^{2}} \epsilon=3 \frac{\dot{R}^{2}+k c^{2}}{R^{2}}, \frac{8 \pi G}{c^{2}} p=-\frac{2 R \ddot{R}+\dot{R}^{2}+k c^{2}}{R^{2}}
$$

where dots denote differentiation with respect to time. These are the well-known cosmological equations, and they are often written in the alternative form

$$
\begin{aligned}
& \frac{d}{d t}\left(\epsilon R^{3}\right)+p \frac{d R^{3}}{d t}=0 \\
& \dot{R}^{2}=\frac{8 \pi G}{3} \rho R^{2}-k c^{2}
\end{aligned}
$$

where $\rho=\epsilon / c^{2}$ is the mass density.

\section{A PARTITIONED UNIVERSE}

Let the universe be divided into cells, of volume $V$, with comoving imaginary partitions that are perfectly reflecting for incident particles and radiation 6 . In 
the actual universe each cell must be at least as large as $U$ to contain average conditions, whereas in an idealized universe $\mathrm{V}$ has in principle no lower limit. The presence of the non-absorbing, massless partitions in no way affects either the properties of the universe or the internal state of the cells. The cosmological equations apply to an idealized universe and in seeking an alternative derivation of these equations we must adopt the same idealizations. It follows that the cells, no matter how small they are made, have at each instant of cosmic time contents which are in an identical state.

If now all partitions are removed except for the boundaries of a given cell, an observer inside this cell discovers conditions identical with those discovered by an observer anywhere outside. The internal observer, although isolated, is in no ways handicapped in his study of the universe (Section VI). For example, owing to the repeated reflection of photons by the expanding walls, he observes red-shifted radiation sources in the same way as the external observer. Provided his boundary conditions are properly maintained it will have no effect if the rest of the universe ceases to exist.

The internal state of a cell is independent of the volume chosen and therefore we are free to consider a cell of an infinitesimally small volume. As $V \rightarrow 0$, the metric of the cell becomes flat, and the internal state remains unchanged if the cell is isolated and embedded in the flat metric of special relativity. The cell remains a faithful representation of the idealized universe and can be regarded as a microcosmic model.

\section{THE MICROCOSMIC MODEL}

The notion of a vanishingly small microcosm of the universe embedded in a flat metric is no more than an interpretation of the cosmological principle applied to a perfect fluid. The microcosmic model, however, allows us to deal with a range of comological problems without resorting to general relativity.

Validity of the Newtonian Approximations

We assume for convenience that the cell is spherical and has a radius a. We consider always a cell of infinitesimal radius such that the metric is flat. The comoving coordinates of an element of fluid in the cell are

$$
\mathbf{r}=\mathbf{S}(\mathbf{t}) \mathbf{r}_{0}, \theta=\theta_{0}, \phi=\phi_{0}
$$


where $r$ is the distance from the center of the cell, $r_{0}$ is the distance at time $t_{0}$, and $S$ is a function of time with $S\left(t_{0}\right)=1$. A cell possessing uniform properties preserves its uniformity as it dilates or contracts. The velocity of a fluid element is

$$
\mathbf{v}=\dot{\mathbf{S}} \mathbf{r}_{0}
$$

From general relativity theory the metric in the weak field approximation is

$$
d s^{2}=d t^{2}\left(c^{2}+2 \psi\right)-d r^{2}-r^{2}\left(d \theta^{2}+\sin ^{2} \theta d \phi^{2}\right)
$$

for $v^{2} \ll c^{2}, 2|\psi| \ll c^{2}$, where $\psi$ is the gravitational potential. Hence the metric is flat when

$$
\mathrm{v}_{\mathrm{r}=\mathrm{a}}^{2}=\frac{\dot{\mathrm{S}}^{2}}{\mathrm{~S}^{2}} \mathrm{a}^{2} \rightarrow 0, \psi_{\mathrm{r}=\mathrm{a}} \sim \mathbf{G} \rho \mathrm{a}^{2} \rightarrow 0
$$

and these conditions can always be achieved by selecting a cell of sufficiently small radius a. Thus as a $\rightarrow 0$ (this does not mean that a cell of initially finite radius shrinks to zero as this would violate the conditions (Equation 9), but means that a cell of vanishingly small radius is chosen and isolated from the universe), the geodesic equations of the fluid elements are accurately given by the Newtonian equations of motion:

$$
\frac{\mathrm{d} \mathbf{v}}{\mathrm{dt}}=-\nabla \psi, \text { or: } \quad \ddot{\mathbf{S}} \mathbf{r}_{0}=-\frac{\partial \psi}{\partial \mathbf{r}}
$$

The conditions (Equation 9) for a flat metric also justify the use of Poisson's equation (for example, Tolman, p. 200). However, the sources of the gravitational field have so far not been specified. Although the Newtonian approximations apply quite accurately to the fluid motion we are still working within a special relativity framework and therefore energy has an equivalent mass. The ratio of the total kinetic energy to $\in V$ is of the order $\dot{S}^{2} a^{2} / s^{2}$, and the ratio of the gravitational potential energy to $\in \mathrm{V}$ is of the order $\mathbf{G} \rho \mathbf{a}^{2}$, and according to (Equation 9) the equivalent mass contribution of both the kinetic and potential energies is vanishingly small. 


\section{Energy Equation}

From the first law of thermodynamics, $d E+p d V=0$, where $d E$ is the increase in the internal energy of the cell, pdV is the work done in expanding against pressure $\mathrm{p}$, and $\delta \mathrm{Q}=0$ because no heat crosses the cell boundary. The energy in the cell is $\mathrm{E}=\epsilon \mathrm{V}$, and therefore the adiabatic equation is

$$
\frac{d}{d t}\left(\epsilon S^{3}\right)+p \frac{d S^{3}}{d t}=0
$$

since $V \propto S^{3}$. Equation (11a) or its alternative form

$$
\frac{\partial \rho}{\partial \mathbf{t}}+\operatorname{div} \underset{\sim}{\sim}\left(\rho+\mathbf{p} / \mathbf{c}^{2}\right)=0
$$

can be obtained in special relativity using $\mathbf{T}_{\mu, \nu}^{\nu}=0(\mu=4)$ and $\mathrm{v} / \mathrm{c} \rightarrow 0$. Equation (11a) does not imply that entropy is necessarily constant. Variations in the composition of the fluid can be associated with entropy changes (Tolman?, Chapter IX).

\section{Boundary Stresses}

Outside the microcosmic cell the pressure is zero and the sphericalboundary, unlike the imaginary partitions in an idealized universe, is now real in the sense that it must exert an inwardly directed force to maintain the cell in a quasi-static state. This boundary force contributes a stress-energy to the total energy of the cell. In some respects the cell is analogous to a soap bubble in a vacuum; the surface stresses contribute an energy of $3 \mathrm{pV}$. The relativistic form of the virial theorem is

$$
\Sigma\left({\underset{\sim}{p}}^{2} \mathrm{~m}^{-1}+\underset{\sim}{\mathrm{r}} \cdot \underset{\sim}{\mathrm{F}}\right)=\int \mathrm{prd} \sigma
$$

where the summation is over all particles in $\mathrm{V}$, of momentum $\mathrm{p}$ and mass $\mathrm{m}$ interacting with each other through forces $\underset{\sim}{\mathrm{F}}$, and the surface integrăl, equal to $3 \mathrm{pV}$, is the energy of the boundary stresses. The virial theorem is commonly used for evaluating the kinetic and interaction energies of an enclosed gas in terms of the boundary stress-energy (for example, Jeans ${ }^{8}$ ), and this stress-energy is then excluded from the kinetic properties of the fluid since it exists merely to 
preserve the fluid in an isolated, quiescent state. When the pressure vanishes at the boundary, as for a star, the left hand side of Equation (12) contains a term

$$
-\int \underset{\sim}{r} \cdot \nabla_{p d V}=3 \int p d V
$$

and this stress-energy is equal to the negative gravitational potential energy 9 . In the microcosmic model the fluid is not gravitationally bound but is confined by a system of stresses that allow the density and pressure to remain uniform. Either we imagine these stresses applied to a single spherical boundary or to an arrangement of partitions within the cell; in any event, the stresses contribute $3 \mathrm{pV}$ to the energy of the cell or $3 \mathrm{pV} / \mathrm{c}^{2}$ to its mass. If these stresses are neglected the fluid will disperse because of the random motions of its particles and the essential properties of the model will be lost.

The mass of the microcosmic cell is $\left(\rho+3 \mathrm{p} / \mathrm{c}^{2}\right) \mathrm{V}$. Using general relativity theory, Tolman ${ }^{7}$, p. 235 derives a similar expression for the energy of a quiescent mass, and Whittaker ${ }^{10}$ shows that in effect $\rho+3 \mathrm{p} / \mathrm{c}^{2}$ is the density of the gravitational mass.

Although the fluid velocity is small the individual particles composing the fluid may have relativistic speeds; the stress-energy contribution is then quite important. To suppose that the stress-energy resides solely in a spherical boundary, as in the case of a soap bubble, creates the anomaly that the boundary and the adjacent fluid have different equations of motion. Hence we must suppose that in addition to the spherical boundary there is an indefinitely large number of internal partitions and the stress-energy is uniformly distributed and has a value of $3 p$ per unit volume.

Poisson's equation is therefore

$$
\nabla^{2} \psi=4 \pi \mathbf{G}\left(\rho+3 \mathbf{p} / \mathbf{c}^{2}\right)
$$

Equations of Motion

Poisson's equation and the equations of energy and motion are

$$
\nabla^{2} \psi=4 \pi \mathbf{G}\left(\rho+3 \mathbf{p} / \mathrm{c}^{2}\right)
$$




$$
\begin{gathered}
\frac{\partial \rho}{\partial \mathrm{t}}=-\operatorname{div} \underset{\sim}{v}\left(\rho+\mathrm{p} / \mathrm{c}^{2}\right) \\
\frac{\mathrm{d} \underset{\sim}{\mathrm{dt}}}{\mathrm{v}}=-\nabla \psi
\end{gathered}
$$

Since $\underset{\sim}{\mathrm{v}}=\underset{\sim}{\dot{\mathrm{S}}} \mathrm{r}_{0}$, the equation of motion becomes

$$
\ddot{\mathrm{S}}=-\frac{4 \pi \mathrm{G}}{3}(\rho+3 \mathbf{p c}-2) \mathrm{S}=\frac{4 \pi \mathrm{G}}{3} \frac{\mathrm{d}}{\mathrm{dS}}\left(\rho \mathrm{S}^{2}\right)
$$

using Equation (13) and Equation (11a, or b). Integrating Equation (14), we find

$$
\dot{\mathrm{S}}^{2}=\frac{8 \pi \mathrm{G}}{3} \rho \mathrm{S}^{2}-\mathrm{C}
$$

where $\mathrm{C}$ is a constant for all fluid elements $\mathrm{S}$ is arbitrary within a constant multiplying factor depending on the time $t_{0}$ chosen to satisfy $S\left(t_{0}\right)=1$. By redefining $S$ such that $S(t)=R(t)|C|^{1 / 2} c^{-1}, S\left(t_{0}\right)=|C|^{1 / 2} c^{-1}$, Equation (15) becomes

$$
\dot{\mathrm{R}}^{2}=\frac{8 \pi \mathrm{G}}{3} \rho \mathrm{R}^{2}-\mathrm{kc}^{2}
$$

where $k=0, \pm 1$. This is the integrated equation of motion of the microcosmic model, and because it applies for all infinitesimally small cells it is also true for the idealized universe. Poisson's equation (13) cannot be transferred from the microcosmic model, and the only surviving equation (11a, or b) applicable to all cells and also true for the idealized universe is

$$
\frac{d}{d t}\left(\epsilon R^{3}\right)+p \frac{d R^{3}}{d t}=0
$$

Equations (16) and (17) are the cosmological equations $(6 \mathrm{a}, \mathrm{b})$ and have been derived by means of the microcosmic model without general relativity theory. 


\section{NEWTONIAN COSMOLOGY}

The equations used in Newtonian cosmology $1,11,12$ are Equations (10), (11) and (13) with the pressure equal to zero. If the gravitational force and velocity fields are isotropic for a given inertial observer, they are also isotropic for all non-inertial observers moving with the fluid 3,13 . The fluid elements have velocities less than, equal to, or greater than the escape velocity, and their trajectories are respectively elliptical $(k=+1)$, parabolic $(k=0)$ or hyperbolic $(k=-1)$, corresponding to elliptical, flat, or hyperbolic space. Newtonian cosmology provides an admirable description of the contemporary universe in which the pressure is relatively small. Callan, Dicke, and Peebles ${ }^{14}$ point out that the Newtonian treatment is a perfectly correct method. They consider a spherical volume of the universe sufficiently small to justify the Newtonian approximations; they do not isolate this spherical element and embed it in flat space as in the microcosmic model.

Consider two observers A and B comoving with the fluid. Let A project a test particle $\alpha$ towards $B$, and let $B$ project a test particle $\beta$ towards $A$ at the same proper speed and at the same instant of cosmic time. In an isotropic and homogeneous universe $\alpha$ must arrive at $B$ at the same instant and with the same proper speed as $\beta$ arrives at $A$. Let us now suppose that $A$ and $B$ are both centers of isotropic gravitational fields. Then $A$ will expect $\alpha$ to arrive at $B$ later than $\beta$ arrives at $A$; and vice versa, $B$ will expect $\beta$ to arrive at $A$ later than $a$ arrives at $B$. Both cannot be correct, and the concept of a gravitational field necessarily implies that A and B are strictly not equivalent. In Newtonian cosmology this loss of equivalence is expressed by the fact that all observers are non-inertial with respect to each other.

A possible objection to Newtonian cosmology is the ambiguous nature of the gravitational field in a uniform unbounded fluid. Layzer ${ }^{2}$ attempts to avoid this difficulty by considering a spherical volume of a pressure-free universe, of arbitrary size and embedded in empty space, and finds it necessary to use general relativity theory. (The microcosmic model is in fact a combination of the ideas of Dicke et al. and Layzer.) $\mathrm{McCrea}^{3}$ avoids the difficulty with the suggestion that the Newtonian universe is regarded as a bounded but arbitrarily large system. For a closed boundary Dirichlet conditions are sufficient to establish everywhere a unique gravitational potential; moreover, if the boundary is spherical, it is reasonable to assume that its center is inertial. Using Newtonian theory, the cosmological equations are derived self-consistently and most simply for the central observer, and the validity of the equations for all observers is deduced from kinematic considerations. Any non-central observer, retaining the same boundary conditions, can derive identical equations from Newtonian theory if he allows for his non-inertial framework. This implies that such an observer 
is aware of the locations of a unique inertial center from which all matter in the universe is diverging.

In Newtonian cosmology the universe is spatially bounded and therefore contains a unique central observer; this conclusion seems inescapable. Such a universe fails to conform with the mediaeval form of the cosmological principle 15 : "the fabric of the world" has|"its center everywhere and its circumference nowhere". Many may think that this is of no great consequence.

\section{DISCUSSION}

The usual idealizations employed in cosmology lead naturally to the idea of a partitioned universe containing infinitesimally small cells. The walls of each cell are perfectly smooth and reflecting and an observer inside any cell perceives conditions identical with those perceived in the absence of the partitions. If his cell contains discrete sources of radiation, he observes, because of multiple reflections, an extended, expanding, and apparently unbounded universe of discrete sources. To this multiple-image universe he applies the cosmological principle and deduces that the universe has a uniform metric given by Equation (1). If he is aware of the reflecting walls he can use periodic boundary conditions for calculating the red-shift, and for a highly relativistic particle, photon, or neutrino, assert that

$$
\frac{\lambda}{\lambda}\left(\begin{array}{l}
\left(t_{2}\right) \\
\left(t_{1}\right)
\end{array}=\frac{R}{R}\left(\begin{array}{l}
\left.t_{2}\right) \\
\left(t_{1}\right)
\end{array}\right.\right.
$$

and the red-shift is

$$
\frac{\delta \lambda}{\lambda_{1}}=\frac{R\left(t_{2}\right)-R\left(t_{1}\right)}{R\left(t_{1}\right)}
$$

where $\lambda$ is the wavelength. Or, using special relativity, the total coordinate path length is

$$
\int d\left(x^{2}+y^{2}+z^{2}\right)^{3 / 2}=c \int \frac{d t}{R(t)}
$$


- and for two light rays emitted by the same source at times $t_{1}$ and $t_{1}+d t_{1}$, and received at $t_{2}$ and $t_{2}+d_{2}$, respectively, the coordinate path length is invariant and therefore

$$
\frac{d t_{2}}{R\left(t_{2}\right)}=\frac{d t_{1}}{R\left(t_{1}\right)} \text {, }
$$

in agreement with Equation (18). Or, ignoring the reflecting walls and using the general line-element (1) for the multiple-image universe, he again derives the red-shift equations (18), and furthermore, deduces a luminosity-distance and the distances by volume and apparent size, and finally uses general relativity as the rational theory to obtain the cosmological equations.

But an internal observer need not use general relativity theory to obtain the cosmological equations if he supposes that his infinitesimally small cell terminates at its boundary in flat, empty space. This is the microcosmic model of the universe. Provided the fluid contained in the cell is pressure-free, the Newtonian treatment is valid and leads to perfectly correct results. When, however, the pressure is not zero, stresses must be incorporated in the cell to maintain it in a quasi-static state. If the pressure is large the individual particle motions and the interacting fields require special relativity theory for their treatment. From the point of view of the microcosmic model the structural stresses needed for isolation in flat space increase the energy of the cell, and the mass-equivalent is an additional source of the gravitational field. When this increased gravitational field is used in the Newtonian equation of motion for the fluid elements we derive, with the aid of the first law of thermodynamics, the cosmological equations.

As in the case of Newtonian cosmology, the microcosmic model gives a physical interpretation of the cosmological equations. By focusing attention on a representative infinitesimally small element of the universe, it reveals and exploits the far-reaching nature of the idealizations on which cosmology is founded. 


\section{RE FERENCES}

1. W. H. McCrea and E. A. Milne, Quart. J. Math. $\underset{\sim}{5}, 73$ (1934).

2. D. Layzer, Astron. J., 59, 268 (1954).

3. W. H. McCrea, Astron. J., $\underset{\sim}{60}, 271$ (1955).

4. H. Bondi, "Cosmology", p. 78, 2nd Ed., Cambridge University Press, Cambridge $\overline{(1960)}$.

5. W. H. McCrea, Proc. Roy. Soc. A 206, 562 (1951).

6. E. R. Harrison, Nature 204, 271 (1964); Monthly Notices of the Roy. Astron. Soc. (to be published).

7. R. C. Tolman, "Relativity, Thermodynamics and Cosmology". Clarendon Press, Oxford, (1934).

8. J. Jeans, "Kinetic Theory of Gases", p. 70, Cambridge University Press, Cambridge, (1952).

9. S. Chandrasekhar, "Stellar Structure", p. 66, University of Chicago Press, Chicago, (1939).

10. E. T. Whittaker, Proc. Roy. Soc. A 149,384 (1935).

11. E. A. Milne, Quart. J. Math. $\underset{\sim}{5}, 64$ (1934).

12. W. H. McCrea, Reports on Progress in Physics 16, p. 321, London Physical Society, (1953).

13. G. C. McVittie, "General Relativity and Cosmology", Chap. VII and p. 192, Chapman and Hall, London, (1956).

14. C. Callan, R. H. Dicke, and P. J. E. Peebles, Am. J. Phys. 33, 105 (1965).

15. Nicholas Cusanus, "Of Learned Ignorance", p. 102, translated by G. Heron, London, (1954). 